\title{
Laborroboter im Dienste rationeller Klebstoffentwicklung
}

Ds.

ie Jowat AG setzt als erster Klebstoffher-

steller weltweit eine Bosch Compact Lab

sätzen zu reduzieren und dabei die im Rahmen der statistischen Versuchsplanung notwendige Station (CLS) für die Entwicklung neuer Dispersionsklebstoffe ein.

Bei der Entwicklung neuer Klebstoffrezepturen sind viele Parameter von erheblichem Einfluss auf die späteren Leistungseigenschaften des Produktes. Sollen die Kundenanforderungen hinsichtlich der Kleb- und Verarbeitungseigenschaften des Klebstoffes punktgenau getroffen werden, bedarf es häufig vieler Versuchsreihen, um die Rohstoffkomponenten in ihrem komplexen Wirkgefüge genauestens aufeinander abzustimmen.

Um den Anteil der Arbeitszeit des Laborpersonals an den sich wiederholenden Prozessen im Rahmen der Mischung von VersuchsanAnzahl der Versuchsreihen zu erhöhen, wurde das CLS angeschafft. Während es vollautomatisch die komplexen Dispersionsformulierungen mischt und rührt, können sich die Mitarbeiter- und Mitarbeiterinnen der Entwicklungsabteilungen bereits der Prüfung und Charakterisierung der nächsten innovativen Klebstoffrezepturen zuwenden. Zukünftig soll ein solcher Roboter auch für Versuchsreihen in Hotmeltsystemen zum Einsatz kommen.

Weitere Infos: Jowat AG, D-32758 Detmold, www.jowat.de

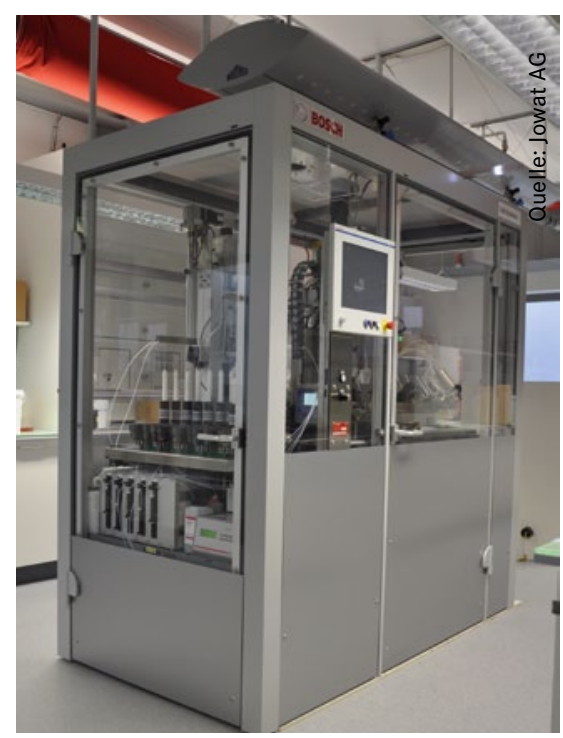

Um die Zeit für die Entwicklung kundenspezifischer Dispersionsklebstoffe zu verkürzen, arbeitet Jowat jetzt mit dem Laborroboter Bosch Compact Lab Station (CLS)

\section{Nitrilhandschuhe: Lange Durchbruchzeiten im Test bestätigt}

D ie Nitrilhandschuhreihe mit dem Markennamen TouchNTuff von Ansell Ltd. erzielte in Tests des belgischen Forschungsinsdurch ein weiches Tragegefühl, eine große Fingerbeweglichkeit tituts Centexbel durchgehend wesentlich längere Durchbruchzeiten bei allgemein verwendeten Laborchemikalien (z.B. Isopropanol und Xylen) als vergleichbare Handschuhprodukte anderer Hersteller.

Die Durchbruchzeit beschreibt die Zeitdauer (in Minuten), die eine Chemikalie für das Durchdringen eines Handschuhs benötigt. Dies kann einen Hinweis darauf geben, über welchen zu erwartenden Zeitraum ein Handschuh eine wirksame Chemikalien- und Flüssigkeitsbarriere bietet, wenn er vollständig in die Chemikalie eingetaucht wird.

Die in zwei Ausführungen erhältlichen Handschuhreihe schützen die Hände in Arbeitsbereichen, in denen die Gefahr besteht, mit Spritzern von gefährlichen Chemikalien in Kontakt zu kommen. Die Materialbasis der Handschuhe ist eine spezifische Nitrilmischung, die sich neben einer hohen Schutzleistung auch sowie einen sehr guten Trockengriff bei schwierig zu handhabenden Materialien (z.B. Glas) auszeichnet.

Die Handschuhe stehen in Kartons à 100 Stück zur Verfügung. Weitere Infos: www.ansell.eu

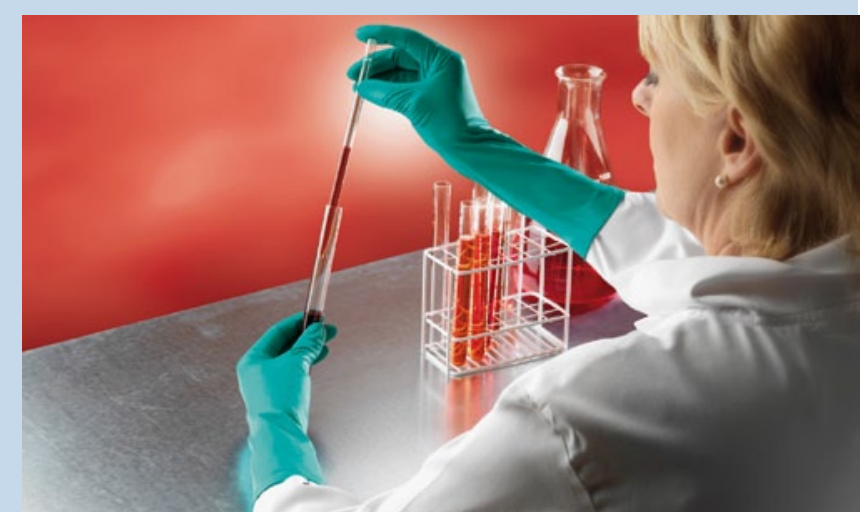

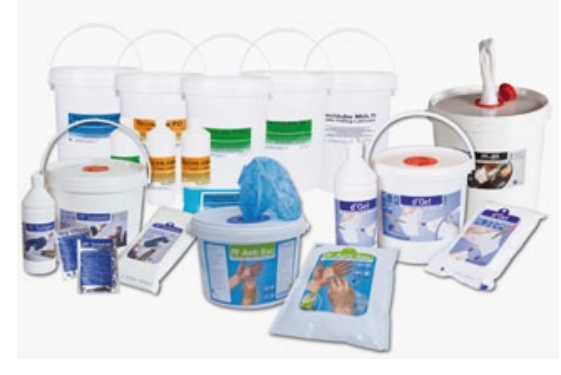

\section{Umweltfreundlich reinigen}

D as irische Unternehmen PT Technologies hat wasserbasierende Hochleistungsreinigungsmittel im Programm, mit denen unterschiedlichste Oberflächen von Bitumen, Harzen und anderer Problem-Medien rückstandslos befreit werden können. Sie bieten die Möglichkeit, Metall, Kupfer oder Kunst- stoff zu reinigen, zu pflegen und z. B. fürs Lackieren oder Kleben vorzubereiten.

Zur Verfügung stehen diese Mittel wahlweise als Flüssigkeit, Gel oder in Form von Tüchern.

Weitere Infos: www.pttechnologies.com, Michael.zickler@pttechnologies.com 